\title{
An Implicit Correlation Method for Cross-Correlation Sampling, with MCNPX-PoliMi Validation
}

\author{
Matthew J. Marcath,* Shaun D. Clarke, and Brian M. Wieger \\ University of Michigan, 2355 Bonisteel Boulevard, Ann Arbor, Michigan 48109 \\ Enrico Padovani \\ Politecnico di Milano, Department of Nuclear Engineering, Via Ponzio 34/3 20133, Milano, Italy \\ and \\ Edward W. Larsen and Sara A. Pozzi \\ University of Michigan, 2355 Bonisteel Boulevard, Ann Arbor, Michigan 48109
}

Received July 24, 2014

Accepted December 27, 2014

http://dx.doi.org/10.13182/NSE14-89

\begin{abstract}
Monte Carlo particle transport codes used to model detector responses are traditionally run in analog mode. However, analog simulations of cross-correlation measurements are extremely timeconsuming because the probability of coincident detection is small, approximately equal to the product of the probabilities of a single detection in each detector. The new implicit correlation method described here increases the number of correlated event scores, thereby reducing variance and required computation times. The cost of the implicit correlation method is comparable to the cost of simulating single-event detection for the lowest absolute detector efficiency in the problem. The new method is especially useful in the nuclear nonproliferation and safeguards fields for simulating correlation measurements of shielded special nuclear material.

The new method was implemented in MCNPX-PoliMi for neutron-neutron cross-correlations with a ${ }^{252} \mathrm{Cf}$ spontaneous fission source measured by 14 detectors at various angles. The method demonstrated good agreement with analog simulation and reference measurement results. Small differences between nonanalog and analog cross-correlation distributions are attributed to discretization errors that are often not present in practical applications. Improvement in the figure of merit was greater than a factor of $100 \mathrm{in}$ all tested cases.
\end{abstract}

\section{INTRODUCTION}

Coincidence measurements are useful to detect and distinguish special nuclear material in the nuclear nonproliferation and safeguards fields. A coincidence measurement counts the instances when at least two radiation detections occur in different detectors within a specified window of time. The time between coincident detections is of particular interest because it characterizes

*E-mail: mmarcath@umich.edu radiation energy spectra for correlated emissions. A histogram of time differences is a coincidence-delay curve or cross-correlation distribution. Special nuclear material consists of fissile material that emits timecorrelated, measurable neutrons from fission events. Organic scintillator detectors are capable of detecting and distinguishing fast neutrons and gamma rays through pulse shape discrimination. ${ }^{1}$ These scintillators have good timing properties and are often used to characterize neutron energy spectra. With pulse shape discrimination and good timing properties, organic scintillators are well 
suited for detecting correlated events and thus for distinguishing special nuclear material from nonfissile radiation sources. ${ }^{2-5}$

The neutrons and photons emitted by a fission event are time correlated, and the neutrons have initial directions of flight that are also correlated. Consequently, accurate coincidence measurement simulations must account for the initial directions of flight; fission event sampling is analog, to simulate properly most coincidence measurements. Currently, Monte Carlo particle transport codes employed for the design and prediction of crosscorrelation measurements using organic scintillators require fully analog simulations to model detector responses accurately. Variance reduction techniques that appropriately model detector response have been developed, such as geometry splitting, ${ }^{6}$ but these techniques still require at least two detections in a fission event for a coincidence.

Unfortunately, the probability of detecting correlated radiation particles in separate detectors is often low, and the presence of shielding exponentially reduces that probability. The computation time associated with simulating low-probability coincidence events scales poorly, approximately with a power equal to the desired number of coincidences (two detections or more) and exponentially with the degree of shielding. Simple measurement geometries for a single detector often require less than an hour of computing-cluster use, whereas a coincidence measurement with shielding could require tens of hours or more to simulate. Such timeintensive simulations, including many practical measurement scenarios, are detrimental to accurate and efficient design innovation.

In a two-detector cross-correlation measurement with a correlated source, e.g., fission, the probability of a coincident detection is approximately the product of the probabilities of detection in each single detector. In this paper, we propose a new implicit correlation Monte Carlo method, which produces cross-correlation events at (roughly) the probability of a single detection in the lowest absolute efficiency detector, effectively reducing the cost of the simulation to that of a single-detector problem. The new method presented here uses event histories in which only a single detection occurs to produce correlation tally information for spontaneous fission point sources in MCNPX-PoliMi. By using singledetection histories to produce correlation tally information, the new method is poised to address computationally intensive correlation measurements, including those that are shielded.

The remainder of this paper is organized as follows. Section II describes the MCNPX-PoliMi code and its built-in spontaneous fission sources. Sections III.A through III.D outline in general terms the method for a coincidence measurement tally with the later sections focusing on the specific application of the method in
MCNPX-PoliMi for a cross-correlation tally. Section IV describes the reference measurement and simulation details. Section V presents results and analysis for the analog, nonanalog, and measurement data with discussion of computation time and speed-up. We conclude with a brief discussion in Sec. VI.

\section{MCNPX-PoliMi}

The PoliMi code extension to the MCNPX Monte Carlo particle transport code was developed to improve the ability to simulate coincidence measurements and subsequent time analyses. ${ }^{7}$ The MCNPX-PoliMi code includes built-in correlations for key isotope fissions, event-by-event tracking, and conservation of energy and momentum event-by-event. ${ }^{7}$

MCNPX-PoliMi has the ability to track and record event information collision-by-collision in specified detector regions. A printed data file, an excerpt of which is shown in Table I, contains a list of collision events that occurred in the specified detector cells. For each collision, key information is included: history number, particle number, particle type, collision type, target nucleus, collision cell, and collision time. Recorded collision information can be used to model nonlinear detector responses accurately event-by-event. Also, the neutron and photon multiplicities for each source fission event can be recorded.

Several spontaneous fission sources are built-in with neutron multiplicity distributions, zero through nine in the case of ${ }^{252} \mathrm{Cf}$ (some sources also have multiplicitydependent energy distributions). ${ }^{8}$ Neutron direction is sampled from an anisotropic distribution, shown in Fig. 1, which is oriented about an isotropically sampled light fission fragment (LFF) direction. Because a majority of the prompt neutrons are emitted from the fully accelerated fission fragments, those neutrons carry momentum from the fission fragments, and thus, an anisotropic neutron angular distribution is observed in the laboratory reference frame. ${ }^{9}$ The neutron angular distribution is dependent on fragment momentum; thus, the angular distribution is dependent on fragment masses. The average angular distribution of neutrons is dependent on the distribution of fragment masses. MCNPX-PoliMi sampling algorithms assume constant fragment masses.

\section{IMPLICIT CORRELATION METHOD}

To illustrate the new implicit correlation method, we present a simple outline of the processes that produce an analog coincidence estimate and a nonanalog coincidence estimate. Consider for simplicity a fission event that emits exactly two neutrons, where the direction and energy of each neutron are independently sampled from the same 


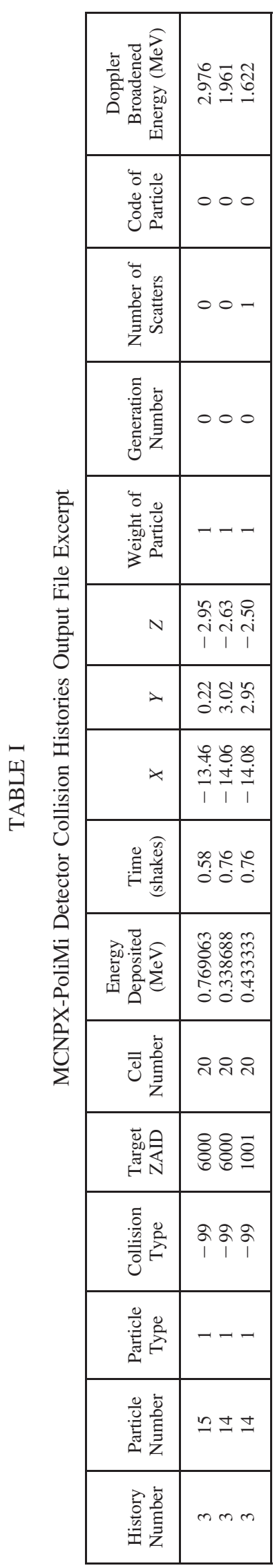

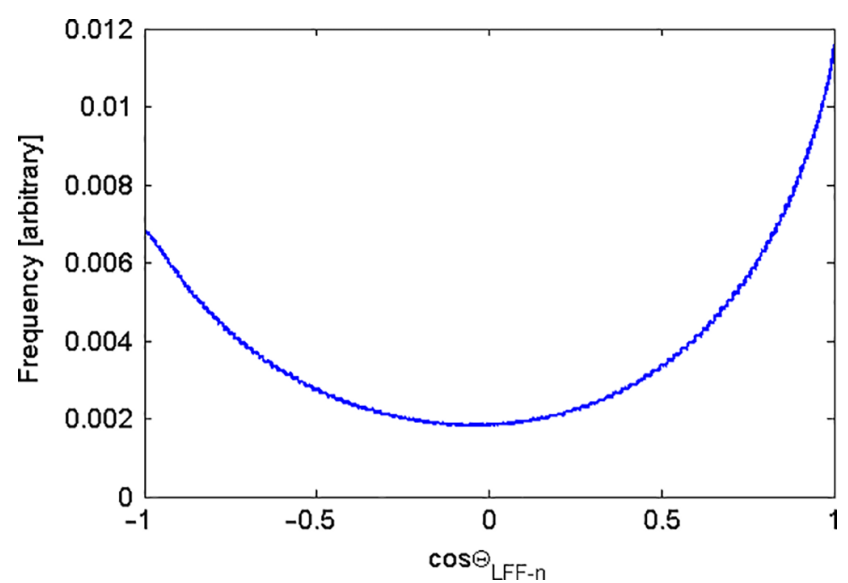

Fig. 1. Neutron angular distribution about the LFF direction. ${ }^{8}$

distribution function. The measurement system consists of the point source and two detectors, labeled $A$ and $B$.

\section{III.A. Analog Coincidence Probability}

The source event emits two neutrons, and each neutron is tracked until death, e.g., absorption, reaching a low energy, or escaping the defined space. If the tracked neutron interacts in detector $A$ or $B$ and deposits sufficient energy, then the interaction is tallied as a detection in that detector; $N_{A}$ or $N_{B}$ is then incremented. Both neutrons are transported from the source event, keeping track of all relevant tallies.

We also consider the following: if one of the two neutrons is detected in detector $A$ and the other neutron is detected in detector $B$, then the coincident detection tally, $N_{A B}$, is incremented.

The source event can be sampled many times (say, $M$ ), with subsequent neutron transport to give tallies $N_{A}$, $N_{B}$, and $N_{A B}$. The probability of detecting a neutron in detector $A$ is estimated as

$$
P_{A} \approx \frac{N_{A}}{2 M}
$$

and in detector $B$ as

$$
P_{B} \approx \frac{N_{B}}{2 M} .
$$

Also, the probability of a coincident detection per source event is estimated as

$$
P_{A B}=\frac{N_{A B}}{M} .
$$

These probabilities will converge as $M \rightarrow \infty$ according to Poisson variance. 


\section{III.B. Nonanalog Coincidence Probability}

Since a coincidence event occurs when (a) the first neutron is detected in detector $A$ and the second in detector $B$ or (b) vice versa, we have the identity

$$
P_{A B}=2 P_{A} P_{B}
$$

Thus, from Eqs. (1) and (2), we have the nonanalog estimate

$$
P_{A B} \approx 2\left(\frac{N_{A}}{2 M}\right)\left(\frac{N_{B}}{2 M}\right)
$$

\section{III.C. Comparison of Error Estimates}

Now we wish to estimate the relative statistical errors in the two estimates of $P_{A B}$ given Eqs. (3) and (5). In the following, we assume the number of fission events $M$ is sufficiently large.

According to the central limit theorem, ${ }^{10}$ we have, with probability 0.954 ,

$$
\left|P_{A B}-\frac{N_{A B}}{M}\right|<\frac{2 \sigma_{A B}}{\sqrt{M}},
$$

where, assuming $P_{A B} \ll 1$,

$$
\sigma_{A B}=\sqrt{P_{A B}\left(1-P_{A B}\right)} \approx \sqrt{P_{A B}} .
$$

Thus, with probability 0.954 ,

relative error in Eq. (3)

$$
\begin{aligned}
& =\frac{1}{P_{A B}}\left|P_{A B}-\frac{N_{A B}}{M}\right|<\frac{2 \sigma_{A B}}{P_{A B} \sqrt{M}} \\
& \approx \frac{2 \sqrt{P_{A B}}}{P_{A B} \sqrt{M}}=\frac{2}{\sqrt{P_{A B} M}} \\
& =\frac{1}{\sqrt{M}} \sqrt{\frac{2}{P_{A} P_{B}}} .
\end{aligned}
$$

Next,

relative error in Eq. (5) $=\frac{1}{P_{A B}}\left|P_{A B}-2\left(\frac{N_{A}}{2 M}\right)\left(\frac{N_{B}}{2 M}\right)\right|$

$$
\begin{aligned}
= & \frac{1}{P_{A B}} \mid P_{A B}-2\left[P_{A}-\left(P_{A}-\frac{N_{A}}{2 M}\right)\right] \\
& \times\left[P_{B}-\left(P_{B}-\frac{N_{B}}{2 M}\right)\right] \mid
\end{aligned}
$$

$$
\begin{aligned}
& =\frac{1}{P_{A B}} \mid P_{A B}-2\left[P_{A} P_{B}-P_{A}\left(P_{B}-\frac{N_{B}}{2 M}\right)\right. \\
& \left.\quad-P_{B}\left(P_{A}-\frac{N_{A}}{2 M}\right)+\left(P_{A}-\frac{N_{A}}{2 M}\right)\left(P_{B}-\frac{N_{B}}{2 M}\right)\right] \mid \\
& =\frac{2}{P_{A B}} \mid P_{A}\left(P_{B}-\frac{N_{B}}{2 M}\right)+P_{B}\left(P_{A}-\frac{N_{A}}{2 M}\right) \\
& \quad-\left(P_{A}-\frac{N_{A}}{2 M}\right)\left(P_{B}-\frac{N_{B}}{2 M}\right) \mid \\
& \quad<\frac{2}{P_{A B}}\left[P_{A}\left|P_{B}-\frac{N_{B}}{2 M}\right|+P_{B}\left|P_{A}-\frac{N_{A}}{2 M}\right|\right. \\
& \left.\quad+\left|P_{A}-\frac{N_{A}}{2 M}\right|\left|P_{B}-\frac{N_{B}}{2 M}\right|\right] .
\end{aligned}
$$

By the central limit theorem, we have, with probability 0.954 ,

$$
\left|P_{A}-\frac{N_{A}}{2 M}\right|<\frac{2 \sigma_{A}}{\sqrt{2 M}} \approx \frac{2 \sqrt{P_{A}}}{\sqrt{2 M}}=\sqrt{\frac{P_{A}}{2 M}}
$$

and

$$
\left|P_{B}-\frac{N_{B}}{2 M}\right|<\frac{2 \sigma_{B}}{\sqrt{2 M}} \approx \frac{2 \sqrt{P_{B}}}{\sqrt{2 M}}=\sqrt{\frac{P_{B}}{2 M}} .
$$

Thus, with probability $(0.954)^{2} \approx 0.9$, Eq. (9) gives the following for $M \gg 1$ :

relative error in Eq. $(5)=\frac{1}{P_{A B}}\left|P_{A B}-2\left(\frac{N_{A}}{2 M}\right)\left(\frac{N_{B}}{2 M}\right)\right|$

$$
<\frac{2}{P_{A B}}\left(P_{A} \sqrt{\frac{P_{B}}{2 M}}+P_{B} \sqrt{\frac{P_{A}}{2 M}}+\sqrt{\frac{P_{A}}{2 M}} \sqrt{\frac{P_{B}}{2 M}}\right),
$$

where the third term is small and ignored:

$$
\begin{aligned}
& \approx \frac{2}{2 P_{A} P_{B}}\left(\frac{P_{A} \sqrt{P_{B}}+P_{B} \sqrt{P_{A}}}{\sqrt{2 M}}\right) \\
& =\sqrt{\frac{2}{M}}\left(\frac{P_{A} \sqrt{P_{B}}+P_{B} \sqrt{P_{A}}}{2 P_{A} P_{B}}\right) \\
& =\frac{1}{\sqrt{M}} \sqrt{\frac{2}{P_{A} P_{B}}}\left(\frac{\sqrt{P_{A}}+\sqrt{P_{B}}}{2}\right) .
\end{aligned}
$$

The right side of the inequality Eq. (10) is always less than the right side of the inequality in Eq. (8) and is much less when $P_{A}$ and $P_{B}$ are small. The confidences for the 
two bounds are different, but nonetheless, when $P_{A}$ and $P_{B}$ are small, the nonanalog estimate of $P_{A B}$ [Eq. (5)] is significantly more efficient than the analog estimate [Eq. (3)].

Similar results can be obtained for sources with multiplicities greater than two, but these will not be derived here. The basic point is that single-detection statistics of neutron interactions with detectors can be used to build estimates of correlated detections in multiple detectors-provided the initial directions and energies of the fission neutrons can be sampled from the same distribution function.

In practice, the initial directions, and sometimes the energies, of neutrons emitted by fission events cannot all be sampled from the same distribution function. This is because the distribution functions for the initial neutron directions and energies can depend on (a) the random direction of flight of the LFF and (b) the multiplicity of the fission event (some PoliMi spontaneous fission sources do not have multiplicity dependent on neutron energy spectra). However, if the LFF directions are binned, then all neutrons that emerge from a fission event - with a specified multiplicity and a LFF traveling in a specified angular bin — can be initialized using the same distribution function. In this way, we apply the ideas described above. All this is discussed in more detailed in the remainder of this paper.

\section{III.D. Another Quantity of Interest: Time Correlation}

Time difference distributions are an information-rich quantity obtained in coincidence measurements. A crosscorrelation distribution simply refers to a histogram of the difference in detection times of the two detections in a coincident event. The histogram may be used to estimate $P_{A B}(\Delta T)$, the probability of a source event with a coincident detection time difference $\Delta T$. To reach a desired uncertainty, an estimate of $P_{A B}(\Delta T)$ requires more histories than an estimate of $P_{A B}$, whereas an estimate of $P_{A B}$ requires more histories than an estimate of $P_{A}$ and $P_{B}$. $P_{A B}$ is useful and straightforward to calculate using indirect or nonanalog methods; the time difference distribution of coincidences $P_{A B}(\Delta T)$ contains spectroscopic information, but it is more difficult to calculate.

Again we consider a source event that emits two neutrons, where each neutron is independently sampled from the same distribution. The measurement system again consists of two detectors, $A$ and $B$, and the source. The source event can be sampled many times, say, $M$, with subsequent neutron transport to give tallies $N_{A}$ and $N_{B}$ as well as a list of detection times $T_{A i}$ and $T_{B j}$, where $i$ and $j$ are integer indices.

The detection times $T_{A i}$ and $T_{B j}$ are independently sampled from identical distributions, since neutrons from each source event are independently sampled from identical distributions. Thus, we can (with no bias) produce a distribution of detection time differences by sampling a time from each detection time list $T_{A i}$ and $T_{B j}$ and subtracting.

\section{III.E. Implicit Correlation Method for Spontaneous Fission}

The new implicit correlation method uses singledetection histories to produce coincidence tally information. Consequently, the number of coincidence scores produced per source event is greater than a corresponding analog simulation. The method utilizes analog fission sampling and particle transport in a nonanalog detector response calculation. To do this, subsets of independent and identically distributed (IID) histories are formed. If a set of simulated fission neutrons in the MCNPX-PoliMi ${ }^{252} \mathrm{Cf}$ model (source option 10) has both the same multiplicity and the same LFF direction in a particular MCNPX-PoliMi problem, those neutrons are IID within that set. Within an IID set of neutron histories, each neutron's initial direction and energy are sampled from the same distributions. In this manner, it is possible to produce IID neutron detection sets by discretizing LFF direction and sorting neutron histories by the source neutron multiplicity.

To simulate a spontaneous fission source, the MCNPX-PoliMi code first samples neutron multiplicity. ${ }^{8}$ Then, neutron energy is sampled from a multiplicityindependent Watt distribution for ${ }^{252} \mathrm{Cf}$; other PoliMi sources have multiplicity-dependent energy sampling. Good agreement is observed in comparisons between simulations using both multiplicity-dependent and independent spectra and measurements. ${ }^{11}$ The multiplicitydependent energy ${ }^{252} \mathrm{Cf}$ source was not used here because the neutron sampling algorithm additionally samples the number of neutrons that are emitted from each fission fragment. The neutron directions are sampled independently from an energy-dependent anisotropic distribution oriented about the LFF direction. Figure 1 shows that neutron directions occur most frequently near the poles of the LFF direction. Thus, the initial directions of spontaneous fission neutrons are correlated by the sampled LFF direction (neutrons are also correlated by multiplicity in multiplicity-dependent energy distributions). However, neutrons from within a particular spontaneous fission history can be independently sampled from identical distributions.

\section{III.F. Removing Correlation in Spontaneous Fission}

A discretized LFF direction distribution can produce results that deviate negligibly from sampling a full distribution. For a set of neutron histories with constant LFF direction and a constant multiplicity, all neutrons in that set are independent and sampled from identical distributions in energy and direction, due to MCNPX- 
PoliMi sampling procedures for ${ }^{252} \mathrm{Cf}$ (source option 10). Thus, for a set of neutron histories with the same LFF direction and multiplicity $m$, one can randomly sample $m$ neutron histories from that set to produce a fission history. The new fission history has been fairly constructed and is of equal weight as any history produced in an analog simulation, given the same LFF direction and multiplicity.

When the LFF direction and multiplicity are fixed for a particular simulation, the neutron histories are correlated only in time and thus are independent. For a correlation measurement scenario with two detectors and independent neutrons, there is also a set of independent detection histories for each detector. Since the neutrons in each set are independent, a coincident event can be fabricated by sampling a history from each detector set. Essentially, the fabricated history is formed by taking a detection event in one detector and, through sampling, implying a coincident detection in the other detector. The implicit coincident tally weight must be found so as not to bias the solution. Tally weight is a function of both detector efficiencies and neutron multiplicity. The estimated probability of detecting a sampled neutron in either detector is calculated from the set of sorted neutron histories; the estimated neutron detection probability can then be used to estimate the probability of coincident detection. The estimated probability of coincident detection is then used to calculate the tally weight. The tally from each bin, where multiplicity and LFF direction were fixed, is multiplied by the appropriate weight and summed to produce the total result of sampling a full multiplicity and LFF direction distribution.

\section{III.G. Tally Weighting}

Neutrons from different fission events are unlikely to cause a coincident detection when the spontaneous fission rate is low in comparison to the detector response time; fissions are well separated in time. Also, with low neutron multiplying sources, coincident detection of neutrons from different fission events is unlikely; therefore, that case is ignored.

For a low spontaneous fission rate, the probability $P_{12}\left(m, p_{1}, p_{2}\right)$ of detecting correlated neutrons for a chosen multiplicity $m$ and chosen LFF direction is a function of the neutron multiplicity and the neutron detection probability for each detector $p_{i}$. The detection probability for each detector $p_{i}$ is multiplicity independent because the neutron energy spectrum is multiplicity independent; however, $p_{i}$ is dependent on LFF direction. The function that describes the probability $P_{12}\left(m, p_{1}, p_{2}\right)$ is a multinomial distribution with three outcomes: detection in detector number one, detection in detector number two, and no detection. ${ }^{12}$ The probability $p_{i}$ is the likelihood that a single neutron sampled from appropriate energy and angular distributions is detected in detector $i$. A coincident detection occurs only when at least one neutron is detected in each detector and a coincident outcome may occur in a number of permutations. Thus, a sum is taken over the probability of each event for $n_{i} \geq 1$. Coincident detections are considered per fission event; coincident neutron detection in a single detector, where $n_{i}>1$, is treated similarly to a $n_{i}=1$ event. A different treatment of higher-order detector events would not significantly influence results because the probability of high-order detections is small when $p_{1}$ and $p_{2}$ are small. The probabilities of a coincident detection $P_{12}$ for multiplicity two or three, and general multiplicity $m$ fission are shown in Eqs. (4), (11), and (12), respectively:

$$
\begin{aligned}
P_{12}\left(3, p_{1}, p_{2}\right)= & 6 p_{1} p_{2}\left(1-p_{1}-p_{2}\right) \\
& +3 p_{1} p_{2}^{2}+3 p_{1}^{2} p_{2}
\end{aligned}
$$

and

$$
\begin{aligned}
P_{12}\left(m, p_{1}, p_{2}\right)= & \sum_{n_{1}=1}^{m-1} \sum_{n_{2}=1}^{m-n_{1}} \frac{m !}{n_{1} ! n_{2} !\left(m-n_{1}-n_{2}\right) !} \\
& \times p_{1}^{n_{1}} p_{2}^{n_{2}}\left(1-p_{1}-p_{2}\right)^{m-n_{1}-n_{2}} .
\end{aligned}
$$

The advantage of the implicit correlation method can be estimated by calculating $P_{12}$ and $p_{i}$ for each detector in the correlation problem. The approximate speed-up will be of the order of the smallest $p_{i}$ divided by $P_{12}$. Further discussion of computation time and speed-up is included in Sec. V.C.

If the detection probabilities $p_{i}$ are known for a set of independent neutrons, then the probability of a coincident detection can be found using Eq. (12). The detection probabilities can be readily calculated for sets of independent particles. The detection probabilities are calculated in separate simulations in which fissions are forced to emit only a single neutron (all other distributions are sampled properly including a multiplicity-independent energy distribution). By emitting only a single neutron per history, detector effects are simplified, and reliable estimates of the detection probabilities can be made. It is possible to use postprocessing to obtain singleneutron detection probabilities, thereby removing the necessity of a separate simulation whose sole purpose is to calculate those probabilities.

For a set of histories of a chosen multiplicity and LFF direction, the expected number of correlated detections $\left\langle N_{12}\right\rangle$ is given by

$$
\left\langle N_{12}\right\rangle=M P_{12}\left(m, p_{1}, p_{2}\right),
$$

where $M$ is the number of fission events. The implicit correlation tally weight must be adjusted to a factor $w$ to give the expected number of coincidences: 


$$
w=\frac{M P_{12}\left(m, p_{1}, p_{2}\right)}{D_{12}},
$$

where $D_{12}$ is the total number of implied pairings for the set.

\section{METHODS}

\section{IV.A. Reference Measurement}

A reference ${ }^{252} \mathrm{Cf}$ cross-correlation measurement was used to compare with analog and nonanalog calculation results. The reference measurement of source 1 is used, which was manufactured in 1994. It has an intensity of 56000 fissions/s and at the time of writing was measured with approximately $14.5 \%$ of fissions from ${ }^{250} \mathrm{Cf}$ and the remainder from ${ }^{252} \mathrm{Cf}$ (Ref. 11). The reference measurement consisted of fourteen $7.62-\mathrm{cm}$-diameter by $7.62-\mathrm{cm}$ EJ-309 organic liquid scintillation detectors in a $40-\mathrm{cm}-$ diameter ring around a ${ }^{252} \mathrm{Cf}$ source on a low-density foam block, shown in Fig. 2. Adjacent detector pairs formed an angle of about $26 \mathrm{deg}$ with the source, but only detector pair angles of 77 and $180 \mathrm{deg}$ are used in this work. The detection system was sensitive to both gamma rays and neutrons; detection events were distinguished using pulse shape discrimination algorithms. Neutronneutron cross-correlation time distributions were recorded for each detector pairing.

\section{IV.B. Simulation Model}

Shown in Fig. 3, the simulation model details each detector, the source, foam block that held the source in position, air, aluminum table, and concrete floor. Other minor geometric aspects of the measurement environment were not modeled. Detector parameters (i.e., threshold,

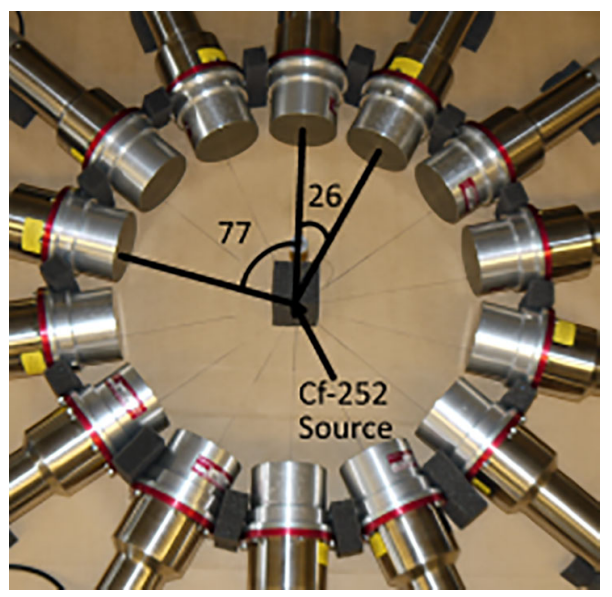

Fig. 2. Photograph of the reference experimental setup. A ring of 14 organic liquid scintillators, each spaced 26 deg from the next, surrounded a ${ }^{252} \mathrm{Cf}$ source.

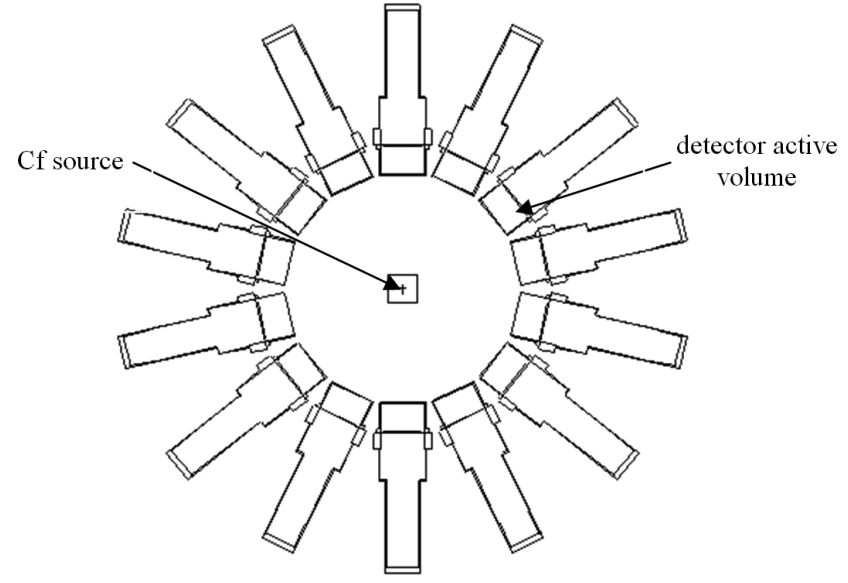

Fig. 3. Top view of the simulation model of the measurement setup.

pulse generation time, and timing resolution) that are the same as those in the reference measurement scenario were used in postprocessing to replicate actual detection effects and responses. The MPPost program was used to postprocess MCNPX-PoliMi data. ${ }^{13}$

The source was approximated as exclusively emitting neutrons and gamma rays through ${ }^{252} \mathrm{Cf}$ spontaneous fissions. Various built-in isotope spontaneous fission options were invoked using the IPOL card in PoliMi. The built-in PoliMi source, $\operatorname{IPOL}(1)=10$, was used because neutrons from spontaneous fission model $\operatorname{IPOL}(1)=1$ are correlated beyond LFF direction and multiplicity. The fission model used in the calculations, samples neutron directions from an anisotropic angular distribution, Fig. 1, determined by the LFF direction and samples neutron energy from a multiplicity-independent Watt distribution.

\section{RESULTS AND ANALYSIS}

The nonanalog results are compared with analog results to verify the implicit correlation method. Also, nonanalog and analog simulation results are compared to the reference measurement of the neutron-neutron crosscorrelation time distribution for two detector pairs, one at $77 \mathrm{deg}$ and one at $180 \mathrm{deg}$, to validate the implicit correlation method implemented in MCNPX-PoliMi.

The analog calculation simulated $1 \times 10^{8}$ fission events. Three MCNPX-PoliMi calculations were run for the nonanalog cases, which each simulated $1 \times 10^{7}$ fission events: two to produce histories for implicit correlation in each angle pair and one to calculate individual detection probabilities.

Nonanalog calculations were performed for a series of LFF binning schemes. Each scheme divided angular space by the azimuthal and polar angles. The binning was done for 90-, 45-, and 22.5-deg angular bin widths, both 
polar and azimuthal; thus, there were 8,32 , and 128 bins, respectively.

\section{V.A. Correlations for 180-deg Detector Pairs}

Figure 4 shows that the neutron-neutron crosscorrelation distribution for a 180-deg detector pair peaks about zero $\Delta T$ with a width of about $10 \mathrm{~ns}$ at half of the peak maximum. Given the timing resolution is of the order of a nanosecond for organic scintillator detectors, a broad peak indicates a wide range of neutron arrival times; thus, a wide range of neutron energies were emitted from the source. The detectors are sensitive to approximately 0.5 through $5 \mathrm{MeV}$ neutron energy deposited (a 40-keVee light output threshold was used in the reference measurement). The flight time over approximately $20 \mathrm{~cm}$ from the source directly to a detector over the sensitive range is approximately 24 and $7 \mathrm{~ns}$. However, some highenergy neutrons that deposit partial energy may arrive earlier than $7 \mathrm{~ns}$.

In each nonanalog case, the general shape of the distribution is similar to the analog shape. The nonanalog case in which the LFF direction is binned on a 90-deg mesh agrees poorly with the analog cross-correlation distribution, but agreement improves greatly with decreasing bin width. The reference measurement data agree well with both the nonanalog and analog simulations. Discrepancies between the simulated and measured data are of the order of those reported in Ref. 11.

Using the analog data as a reference, the relative error between -5 and $5 \mathrm{~ns}$ in the nonanalog MCNPX-PoliMi distributions is less than $25 \%$ in the 90-deg mesh case and less than $10 \%$ in the smaller bin cases. Figure 5 shows that as the bin width decreases, the relative error decreases, as expected. Further discretization of the LFF direction could improve results, but more fission histories would be required to maintain adequate sampling in each bin.

The implicit correlation pairing produces results that are nearer to an isotropic neutron angular distribution when the bin widths are large but approach the true

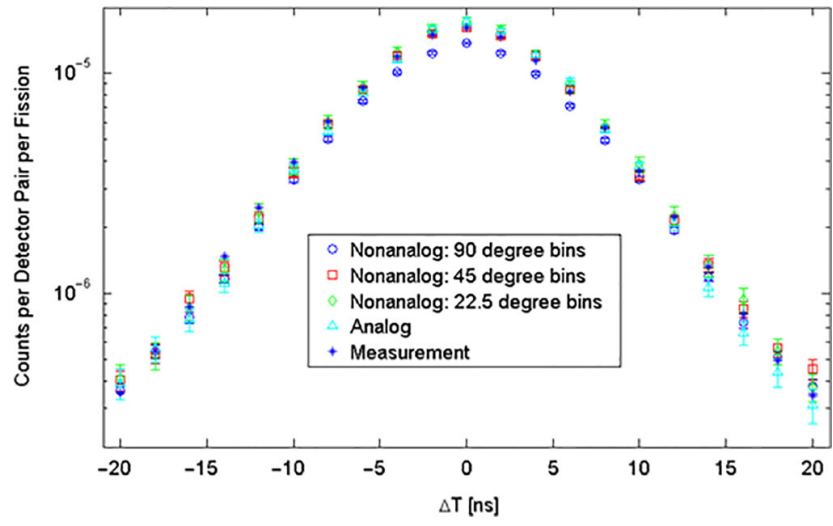

Fig. 4. Neutron-neutron cross-correlation distributions for measurement and simulation of a 180-deg detector pair.

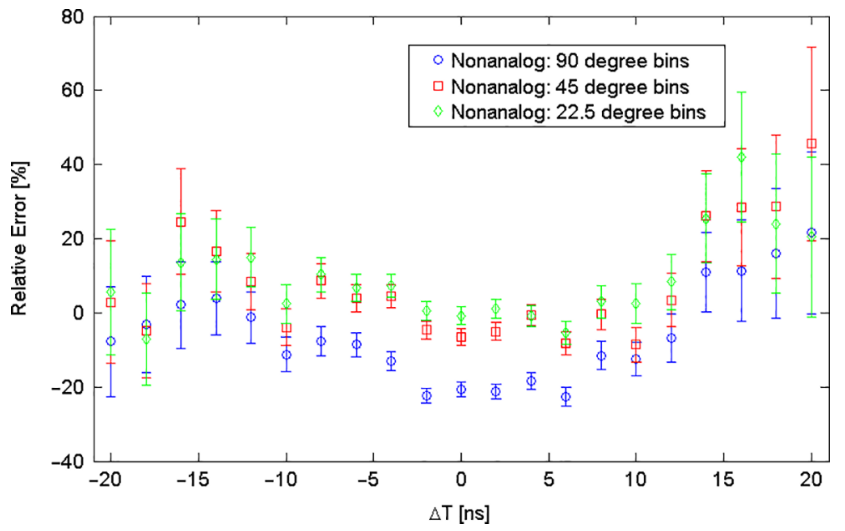

Fig. 5. Relative error between nonanalog and analog neutron-neutron cross-correlation distributions for a 180-deg detector pair.

anisotropic distribution as bin widths narrow. Larger bin widths produce poor agreement with analog calculations in this measurement scenario; however, smaller bin widths tend toward too few detection histories per bin, possibly resulting in undersampling errors. For this calculation, over 1000 history scores occurred in most bins in the 90-deg mesh case, a few hundred in the 45-deg mesh case, and 100 in the 22.5-deg mesh case.

\section{V.B. Correlations for 77-deg Detector Pairs}

Due to the anisotropic neutron distribution about the fission fragment direction, fewer correlated neutronneutron counts are observed for detector pair angles around $90 \mathrm{deg}$ than angles around $180 \mathrm{deg}$. Thus, angular correlation between neutrons observed in the measurement is preserved in the simulation results. The shape of the 77-deg cross-correlation distribution is slightly broader than the 180-deg case because energy is correlated to the angle of emission. Neutrons are more likely to be emitted with lower energy when perpendicular to the LFF direction resulting in higher $\Delta T \mathrm{~s}$, and a broader cross-correlation distribution at detector pairs near $90 \mathrm{deg}$. Additionally, cross-talk is more likely at low detector angles. Cross-talk counts occur when a neutron scatters from one detector to another depositing sufficient energy to cause detections in both; consequently, these counts have a high $\Delta T$ relative to most true two-neutron coincident counts.

In each nonanalog case the general shape of the distribution is similar to the analog, as shown in Fig. 6. The nonanalog case in which the LFF direction is binned on a 90-deg mesh agrees poorly with the analog crosscorrelation distribution, but as expected, agreement improves greatly with decreasing bin width. The reference measurement distribution is broader than the simulated distributions. The disagreement may be due to inaccuracy in the simulation model of fission neutron anisotropy and 


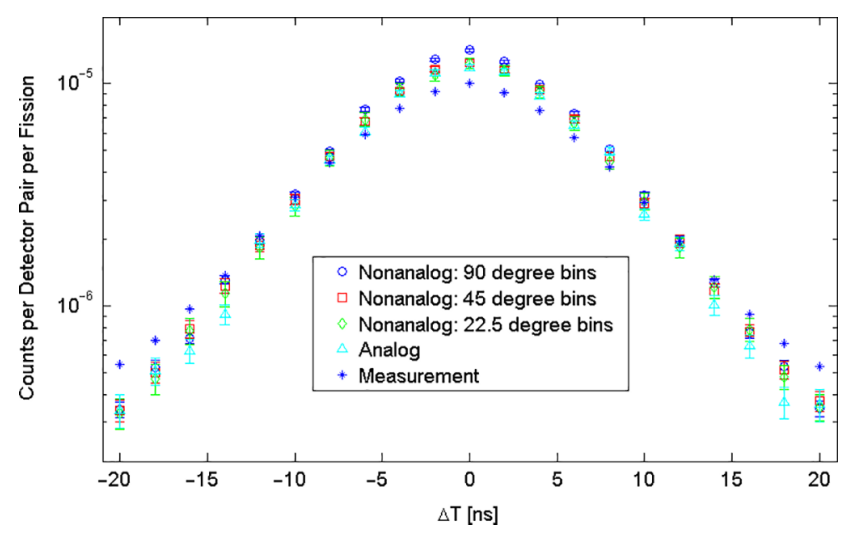

Fig. 6. Neutron-neutron cross-correlation distributions for measurement and simulation of a 77-deg detector pair.

energy about the fission fragment direction. However, discrepancies between the simulated and measured data are of the order of those reported in Ref. 11.

Using the analog data as a reference, the relative error between -5 and $5 \mathrm{~ns}$ in the nonanalog MCNPX-PoliMi distributions is less than $20 \%$ in the $90-$ deg mesh case and about a few percent in the smaller bin cases shown in Fig. 7. As the bin width decreases, the relative error decreases, as expected.

Error between the nonanalog and analog solutions is primarily dependent on the angular distribution of neutrons from the source and not the angle between detectors. In this measurement, neutrons are observed with approximately the same angular distribution of emission from fission; neutrons are unlikely to scatter between the source and detectors. The angular distribution of neutrons in Fig. 1 shows that the distribution changes considerably from the peaks at 1 and -1 to the valley at 0 . The nonanalog solution best captures the anisotropy when the LFF direction is binned on a mesh comparable to changes across the angular distribution. In a 22.5-deg mesh, the observed difference in the probability of neutron emission within a particular bin is at most $25 \%$

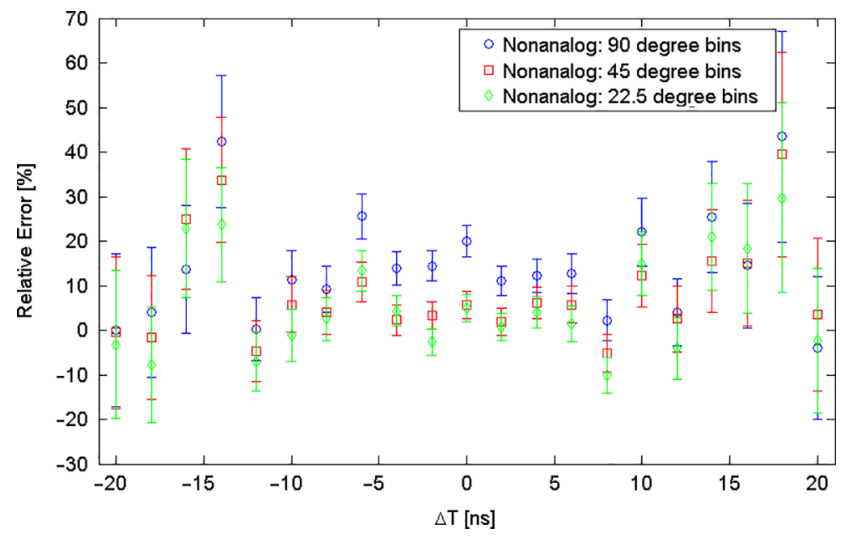

Fig. 7. Relative error between nonanalog and analog neutron-neutron cross-correlation distributions for a 77-deg detector pair. whereas a 90-deg mesh has at most $84 \%$. A measurement with more fission neutron scattering would not require a fine mesh because as neutrons scatter they are observed to be more isotropic. Since the implicit correlation pairing produces results that are nearer to an isotropic neutron angular distribution, as bin widths decrease the nonanalog distribution approaches the analog from above. The number of history scores in each mesh was similar to those described in the previous section.

\section{V.C. Computation Time}

The improvement factor in the simulation variance, a ratio of the nonanalog to analog figure of merits, ${ }^{14}$ was calculated using

$$
F O M=\frac{1}{T R^{2}}
$$

where $T$ is computation time and $R$ is the sample-relative standard deviation of the mean. The mean improvement factor in peak cross-correlation bins ( -10 to $10 \mathrm{~ns}$ ) was found to be greater than 100 in every case.

The improvement factor is substantial because singleneutron detections are used more efficiently than in the analog computation. An analog calculation requires at least two neutron detections for a coincident count whereas in the nonanalog calculation for the majority of histories, single detections are used. Tables II and III show the same trend and the same approximate improvement factors for the two detector angle pairs. As the fragment direction is binned on a finer mesh, relative standard deviation increases slightly because there are fewer scores per angular bin, thereby reducing the improvement factor.

TABLE II

Improvement Factors for 180-deg Detector Pair for Various LFF Angular Bin Widths

\begin{tabular}{|c|c|}
\hline LFF Direction Bin Width (deg) & Improvement Factor \\
\hline 90 & 431 \\
45 & 315 \\
22.5 & 179 \\
\hline
\end{tabular}

TABLE III

Improvement Factors for 77-deg Detector Pair for Various LFF Angular Bin Widths

\begin{tabular}{|c|c|}
\hline LFF Direction Bin Width (deg) & Improvement Factor \\
\hline 90 & 595 \\
45 & 283 \\
22.5 & 144 \\
\hline
\end{tabular}


To estimate the improvement factor, the relative standard deviation is considered the dominant term in Eq. (15); computation time scales approximately linearly with the number of histories. Therefore, the standard deviation of the coincidence probability estimate for the analog and nonanalog calculations is considered. Taking a ratio of the relative error squared [a ratio of Eqs. (8) and (10) squared] gives

$$
\left(\frac{[\text { relative error in Eq. (3) }]}{\text { [relative error in Eq. (5) }]}\right)^{2}=\left(\frac{2}{\sqrt{P_{A}}+\sqrt{P_{B}}}\right)^{2} \text {. }
$$

Equation (16) is a reasonable estimate of the expected improvement factor for the multiplicity two case and a reasonable first-order estimate for higher multiplicities. The probability of neutron detection for an isotropically emitted neutron in the simulated measurement was approximately $3 \times 10^{-3}$. We have from Eq. (16), using $P_{A}=P_{B}=3 \times 10^{-3}$, a ratio of relative standard deviations of approximately 330 . The estimated improvement is of the same order as the observed improvement.

\section{CONCLUSIONS}

The implicit correlation method accurately produces a neutron-neutron cross-correlation distribution, including neutron-neutron angular correlation features, while speeding up convergence time over an analog calculation by over two orders of magnitude. The improvement in computation time is of the order of the probability of a single detection divided by the probability of a correlated detection; this is large in most practical applications. In most cases, discretization errors of a few percent were observed in the peak of the cross-correlation distributions, but those errors would be decreased in practical problems, e.g., a shielded source where scattering reduces neutron anisotropy. Further studies to analyze the implicit correlation method's performance in shielded problems would be useful. Also, further research would seek to expand this method to spatially distributed spontaneous fission sources and induced fission problems.

\section{ACKNOWLEDGMENTS}

This research was performed under appointment to the U.S. Department of Energy Nuclear Nonproliferation International Safeguards Graduate Fellowship Program sponsored by the National Nuclear Security Administration Office of Nonproliferation and International Security. Partial funding for this work was provided by the National Science Foundation and Domestic Nuclear Detection Office/Department of Homeland Security Academic Research Initiative: New Detectors, Electronics, and Algorithms for Fast Neutron Spectroscopy in a Scalable Measurement Platform, under award CMMI 0938909.

\section{REFERENCES}

1. G. F. KNOLL, Radiation Detection and Measurement, John Wiley \& Sons, Hoboken, New Jersey (2010).

2. R. C. RUNKLE et al., "Securing Special Nuclear Material: Recent Advances in Neutron Detection and Their Role in Nonproliferation," J. Appl. Phys., 108, 2 (2010); http:// dx.doi.org/10.1063/1.3503495.

3. L. HOLEWA et al., "Using Neutron Angular Anisotropy Information to Dynamically Determine the Ratio of the $(\alpha, n)$ Rate to Spontaneous Fission Rate for Coincidence Counting Applications," Nucl. Instrum. Methods Phys. Res., Sect. A, 701, 249 (2013); http://dx.doi.org/10.1016/j.nima. 2012.11.026.

4. P. PEERANI et al., "Testing on Novel Neutron Detectors as Alternative to ${ }^{3} \mathrm{He}$ for Security Applications," Nucl. Instrum. Methods Phys. Res., Sect. A, 696, 110 (2012); http://dx.doi.org/ 10.1016/j.nima.2012.07.025.

5. E. C. MILLER et al., "Time-Correlated Pulse-Height Measurements of Low-Multiplying Nuclear Materials," Nucl. Instrum. Methods Phys. Res., Sect. A, 729, 108 (2013); http://dx.doi.org/10.1016/j.nima.2013.06.062.

6. S. PRASAD et al., "Time-Dependent Non-Analog MCNPX-PoliMi Scintillation Detector Response for Neutrons," Trans. Institute of Nuclear Materials Management 53rd Annual Mtg. Orlando, Florida, July 15-19, 2012.

7. S. A. POZZI et al., "MCNP-PoliMi: A Monte Carlo Code for Correlation Measurements," Nucl. Instrum. Methods Phys. Res., Sect. A, 513, 550 (2003); http://dx.doi.org/10.1016/j.nima. 2003.06.012.

8. S. A. POZZI et al., "MCNPX-PoliMi for Nuclear Nonproliferation Applications," Nucl. Instrum. Methods Phys. Res., Sect. A, 694, 119 (2012); http://dx.doi.org/10.1016/j.nima. 2012.07.040.

9. J. RANDRUP and R. VOGT, "Refined Treatment of Angular Momentum in the Event-by-Event Fission Model FREYA," Phys. Rev. C, 89, 044601 (2014); http://dx.doi.org/ 10.1103/PhysRevC.89.044601.

10. A. PAPOULIS, Probability, Random Variables, and Stochastic Processes, McGraw-Hill, New York (1991).

11. S. A. POZZI et al., "Correlated Neutron Emissions from ${ }^{252}$ Cf," Nucl. Sci. Eng., 178, 2, 250 (2014); http://dx.doi.org/ 10.13182/NSE13-96.

12. C. FORBES et al., Statistical Distributions, John Wiley \& Sons, Hoboken, New Jersey (2011).

13. E. C. MILLER et al., "MCNPX-PoliMi Post-Processing Algorithm for Detector Response Simulations," J. Nucl. Mater. Manage., 40, 2, 34 (2012).

14. X-5 MONTE CARLO TEAM, "MCNP-A General NParticle Transport Code, Version 5, Volume I: Overview and Theory," LA-UR-03-1987, Los Alamos National Laboratory (2003). 